Article

\title{
Properties of Functions Involving Struve Function
}

\author{
Jonathan Aaron Azlan Mosiun and Suzeini Abdul Halim * \\ Institute of Mathematical Sciences, Faculty of Science, University of Malaya, Kuala Lumpur 50603, Malaysia; \\ jonathanaaron1992@gmail.com \\ * Correspondence: suzeini@um.edu.my
}

Received: 15 October 2018; Accepted: 4 November 2018; Published: 7 November 2018

Abstract: Let $f(z)=z+\sum_{n=2}^{\infty} a_{n} z^{n}$ and $g_{p, b, c}(z)=z+\sum_{n=2}^{\infty} \frac{\left(-\frac{c}{4}\right)^{n-1}}{\left(\frac{3}{2}\right)_{n-1}(k)_{n-1}} z^{n}$ with $p, b, c \in \mathbb{C}, k=$ $p+\frac{b+2}{2} \neq 0,-1,-2, \ldots$ be two analytic functions in the unit disk $U=\{z:|z|<1\}$. This paper gives conditions so that the function $T_{p, b, c}(z)=(f * g)(z)$, a function associated with the Struve function, is univalent, starlike, or convex in the unit disk.

Keywords: analytic; univalent; Struve function

MSC: $30 C 45$

\section{Introduction}

In light of Louis de Brange using a special function, namely the generalized hypergeometric function, in proving the Bieberbach Conjecture, renewed interest was sparked among the mathematics community in special functions. Following this, many articles were presented in dealing with the geometric properties of different types of special functions including but not limited to generalized hypergeometric function, Gaussian, Kummer hypergeometric functions, Bessel functions, and, most recently, Struve functions [1-9]. Sufficient conditions on the parameters of these special functions were also determined by many authors for them to belong to a certain class of univalent functions [10-20].

Let $\mathcal{A}$ denote the class of analytic functions in the unit disk $U=\{z:|z|<1\}$ of the following form:

$$
f(z)=z+\sum_{n=2}^{\infty} a_{n} z^{n}
$$

and normalized by $f(0)=f^{\prime}(0)-1=0$. Denote $\mathcal{S}$ to be the subclass of $\mathcal{A}$ consisting of univalent functions. Subsequently, denote $\mathcal{S}^{*}$ and $\mathcal{C}$ to be subclasses of function $\mathcal{S}$ which are starlike and convex, respectively, in the unit disk $U$ with the following definitions:

Definition 1. A set $D$ in the plane is said to be starlike with respect to $w_{0}$ an interior point of $D$ if each ray with initial point $w_{0}$ intersects the interior of $D$ in a set that is either a line segment or a ray. If a function $f(z)$ maps $U$ onto a domain that is starlike with respect to $w_{0}$, then we say that $f(z)$ is starlike with respect to $w_{0}$. In the special case that $w_{0}=0$, we say that $f(z)$ is a starlike function.

Definition 2. A set $D$ in the plane is called convex if, for every pair of points $w_{1}$ and $w_{2}$ in the interior of $D$, the line segment joining $w_{1}$ and $w_{2}$ is also in the interior of $D$. If a function $f(z)$ maps $U$ onto a convex domain, then $f(z)$ is called a convex function. 
The analytical definition for the classes of starlike and convex functions are as follows, where $\Re$ denotes the real part of a complex function:

Proposition 1. Let $f \in \mathcal{S}$, then $f \in \mathcal{S}^{*}$ if and only if

$$
\Re\left\{\frac{z f^{\prime}(z)}{f(z)}\right\}>0(z \in U) .
$$

Proposition 2. Let $f \in \mathcal{A}$, then $f \in \mathcal{C}$ if and only if

$$
\Re\left\{1+\frac{z f^{\prime \prime}(z)}{f^{\prime}(z)}\right\}>0(z \in U) .
$$

For more insights on these classes, refer to References [21,22].

According to the Alexander theorem [23], every function $f$ is convex in the unit disk if and only if $z f^{\prime}$ is starlike in the unit disk, i.e., $f \in \mathcal{C} \Leftrightarrow z f^{\prime} \in \mathcal{S}^{*}$.

Given any analytic functions, $f(z)=\sum_{n=0}^{\infty} a_{n} z^{n}$ and $g(z)=\sum_{n=0}^{\infty} b_{n} z^{n}$, define the convolution function of $f$ and $g$, denoted by $f * g$, as

$$
(f * g)(z)=\sum_{n=0}^{\infty} a_{n} b_{n} z^{n} .
$$

Trivially, $f * g$ is analytic and it is sometimes referred to as the Hadamard product of $f$ and $g$ in honour of $J$. Hadamard, where Hadamard used an alternative representation,

$$
(f * g)(z)=\frac{1}{2 \pi i} \int_{|\xi|=\rho} \frac{f\left(\frac{z}{\xi}\right) g(\xi)}{\xi} d \xi, \quad|z|<\xi<1,
$$

to illustrate the convolution.

Firstly, consider the following differential equation [24]:

$$
z^{2} w^{\prime \prime}(z)+z w^{\prime}(z)+\left(z^{2}-p^{2}\right) w(z)=\frac{4\left(\frac{z}{2}\right)^{p+1}}{\sqrt{\pi} \Gamma\left(p+\frac{1}{2}\right)}
$$

where $p \in \mathbb{R}$ and a particular solution for Equation (2) is

$$
H_{p}(z)=\sum_{n=0}^{\infty} \frac{(-1)^{n}}{\Gamma\left(n+\frac{3}{2}\right) \Gamma\left(p+n+\frac{3}{2}\right)}\left(\frac{z}{2}\right)^{2 n+p+1} \quad(z \in \mathbb{C}) .
$$

The function $H_{p}(z)$ is known as the Struve function of order $p$. Next, consider the following differential equation which only differs in the coefficient of $w$ :

$$
z^{2} w^{\prime \prime}(z)+z w^{\prime}(z)-\left(z^{2}+p^{2}\right) w(z)=\frac{4\left(\frac{z}{2}\right)^{p+1}}{\sqrt{\pi} \Gamma\left(p+\frac{1}{2}\right)}
$$

and a particular solution for Equation (3) is

$$
\begin{aligned}
L_{p}(z) & =-i e^{-\frac{i p \pi}{2}} H_{p}(i z) \\
& =\sum_{n=0}^{\infty} \frac{1}{\Gamma\left(n+\frac{3}{2}\right) \Gamma\left(p+n+\frac{3}{2}\right)}\left(\frac{z}{2}\right)^{2 n+p+1}(z \in \mathbb{C}),
\end{aligned}
$$


where $L_{p}(z)$ is known as the modified Struve function of order $p$. Refer to Reference [25] for a more in-depth discussion on the Struve function. Now, consider the differential equation

$$
z^{2} w^{\prime \prime}(z)+b z w^{\prime}(z)+\left[c z^{2}-p^{2}+(1-b) p\right] w(z)=\frac{4\left(\frac{z}{2}\right)^{p+1}}{\sqrt{\pi} \Gamma\left(p+\frac{b}{2}\right)},
$$

where $b, c, p \in \mathbb{C}$. Note that if $b=1$ and $c=1$, then Equation (4) reduces to Equation (2), and if $b=1$ and $c=-1$, then Equation (4) reduces to Equation (3). A particular solution for Equation (4), denoted by $w_{p, b, c}(z)$, is

$$
w_{p, b, c}(z)=\sum_{n=0}^{\infty} \frac{(-1)^{n} c^{n}}{\Gamma\left(n+\frac{3}{2}\right) \Gamma\left(p+n+\frac{b+2}{2}\right)}\left(\frac{z}{2}\right)^{2 n+p+1} \quad(z \in \mathbb{C}) .
$$

Similarly to before, if $b=1$ and $c=1$, then $w_{p, 1,1}(z)=H_{p}(z)$, and if $b=1$ and $c=-1$, then $w_{p, 1,-1}(z)=L_{p}(z)$. This generalization allows the study of $H_{p}(z)$ and $L_{p}(z)$ together. Thus, $w_{p, b, c}(z)$ is identified as the generalized Struve function of order $p$. Although the series representation of $w_{p, b, c}(z)$ is convergent everywhere in $\mathbb{C}$, the function is univalent generally in $U$ [26]. Now, consider the function $u_{p, b, c}(z)$ defined as follows:

$$
u_{p, b, c}(z)=2^{p} \sqrt{\pi} \Gamma\left(p+\frac{b+2}{2}\right) z^{\frac{-p-1}{2}} w_{p, b, c}(\sqrt{z}) .
$$

Utilizing the Pochhammer symbol, $(\gamma)_{n}=\frac{\Gamma(\gamma+n)}{\Gamma(\gamma)}=\gamma(\gamma+1) \ldots(\gamma+n-1)$, the following form of $u_{p, b, c}(z)$ can be written:

$$
u_{p, b, c}(z)=\sum_{n=0}^{\infty} \frac{\left(-\frac{c}{4}\right)^{n}}{\left(\frac{3}{2}\right)_{n}(k)_{n}} z^{n}=b_{0}+b_{1} z+b_{2} z^{2}+\ldots
$$

where $k=p+\frac{b+2}{2} \neq 0,-1,-2, \ldots$ and

$$
b_{n}=\frac{(-1)^{n} c^{n} \Gamma\left(\frac{3}{2}\right) \Gamma(k)}{4^{n} \Gamma\left(n+\frac{3}{2}\right) \Gamma(n+k)}
$$

for $n \geq 0$. The function $u_{p, b, c}$ is analytic in $\mathbb{C}$, and satisfies the condition $u_{p, b, c}(0)=1$, as well as the differential equation

$$
4 z^{2} u^{\prime \prime}(z)+2(2 p+b+3) z u^{\prime}(z)+(c z+2 p b) u(z)=2 p+b .
$$

For more discussion on generalized Struve function, refer to References [26-28].

The function $T_{p, b, c}(z)$ is a convolution of $f \in \mathcal{A}$ and $g_{p, b, c}(z)=z u_{p, b, c}(z)$, i.e.,

$$
T_{p, b, c}(z)=\left(f * g_{p, b, c}\right)(z)=z+\sum_{n=2}^{\infty} \frac{\left(-\frac{c}{4}\right)^{n-1}}{\left(\frac{3}{2}\right)_{n-1}(k)_{n-1}} a_{n} z^{n} \quad(z \in U),
$$

where $p, b, c \in \mathbb{C}$ and $k=p+\frac{b+2}{2}$. In Reference [26], Orhan and Yagmur investigated the geometric properties for the function $g_{p, b, c}(z)$, and this prompts the motivation to seek similar properties for the function $T_{p, b, c}(z)$. The function $T_{p, b, c}$ was first introduced by Raza and Yagmur [29]. As such, this paper studies univalency, starlikeness, and convexity properties of the function $T_{p, b, c}$. 


\section{Preliminaries}

The following preliminary results are needed to prove the results in the next section. These results can be found in References [30-33] respectively except for Lemma 3 which can be found in Reference [16].

Theorem 1. If $f \in \mathcal{S}$, then $\left|a_{n}\right| \leq n$ for $n \geq 2$.

Lemma 1. If $f \in \mathcal{A}$ satisfies the inequality

$$
\left|\frac{z f^{\prime}(z)}{f(z)}-1\right|<M(z \in U),
$$

where $M$ is the solution of the equation $\cos (M)=M$, then $\Re\left\{f^{\prime}(z)\right\}>0$.

Lemma 2. If $f \in \mathcal{A}$ and $\Re\left\{f^{\prime}(z)\right\}>0$, then $f$ is univalent.

Lemma 3. If $f \in \mathcal{A}$ and

$$
\left|\frac{z f^{\prime}(z)}{f(z)}-1\right|^{1-\beta}\left|\frac{z f^{\prime \prime}(z)}{f^{\prime}(z)}\right|^{\beta}<(1-\alpha)^{1-2 \beta}\left(1-\frac{3 \alpha}{2}+\alpha^{2}\right)^{\beta},
$$

for some fixed $\alpha \in\left[0, \frac{1}{2}\right], \beta \geq 0$ and for all $z \in U$, then $f \in \mathcal{S}^{*}(\alpha)$.

Proposition 3. Consider for $x \in \mathbb{R}$, the quartic function $P(x)$ of the form

$$
P(x)=r x^{4}+s x^{3}+t x^{2}+u x+v,
$$

where $r, s, t, u, v \in \mathbb{R}$. Solutions of $P(x)=0$ are given as follows:

$$
\begin{aligned}
& x_{1,2}=-\frac{s}{4 r}-S \pm \frac{1}{2} \sqrt{-4 S^{2}-2 a+\frac{d}{S}} \\
& x_{3,4}=-\frac{s}{4 r}+S \pm \frac{1}{2} \sqrt{-4 S^{2}-2 a-\frac{d}{S}}
\end{aligned}
$$

where $a$ and $d$ are the coefficients of the second and of the first degree, respectively, in the associated depressed quartic

$$
\begin{gathered}
a=\frac{8 r t-3 s^{2}}{8 r^{2}}, \\
d=\frac{s^{3}-4 r s t+8 r^{2} u}{8 r^{3}},
\end{gathered}
$$

and where

$$
\begin{gathered}
S=\frac{1}{2} \sqrt{-\frac{2}{3} a+\frac{1}{3 r}\left(Q+\frac{\Delta_{0}}{Q}\right)}, \\
Q=\sqrt[3]{\frac{\Delta_{1}+\sqrt{\Delta_{1}^{2}-4 \Delta_{0}^{3}}}{2}},
\end{gathered}
$$

with

$$
\begin{gathered}
\Delta_{0}=t^{2}-3 s u+12 r v \\
\Delta_{1}=2 t^{3}+9 s t u+27 s^{2} v+27 r^{2}-72 r t v .
\end{gathered}
$$




\section{Results}

Sufficient conditions for $T_{p, b, c}$ to be univalent, starlike, and convex are shown in the theorems below, respectively.

Theorem 2. Let $f \in \mathcal{A}$ and $T_{p, b, c}(z)$ be defined by Equation (5). If $p, b, c, \in \mathbb{C}, k_{1}=p+\frac{b+2}{2}$ and

$$
\begin{aligned}
k_{1} & >\frac{7 M+2+\beta+\sqrt{\frac{16 M^{3}+(17 \beta+100) M^{2}+(40 \beta+80) M+12 \beta+16-\beta^{3}}{\beta}}}{24 M}|c| \\
& \approx 1.098143352|c|,
\end{aligned}
$$

where $M$ is the solution the equation $\cos (M)=M$ and

$$
\begin{aligned}
\beta & =\sqrt{\frac{1}{3}\left[\frac{49}{\alpha} M^{3}+\left(\frac{28}{\alpha}+17\right) M^{2}+\left(\frac{4}{\alpha}+\alpha+40\right) M+12\right]} \\
& \approx 3.469588501
\end{aligned}
$$

with

$$
\begin{aligned}
\alpha^{3} & =-343 M^{3}-2886 M^{2}-948 M-8+24 \sqrt{3 M} \sqrt{1029 M^{4}+5113 M^{3}+3138 M^{2}+540 M+8} \\
& \approx-28.2795216
\end{aligned}
$$

then $\Re\left\{T_{p, b, c}^{\prime}(z)\right\}>0$ for all $z \in U$.

Proof. Suppose $f \in \mathcal{A}$. Using $\left|z_{1}+z_{2}\right| \leq\left|z_{1}\right|+\left|z_{2}\right|$, the inequalities $\left(\frac{3}{2}\right)_{n} \geq \frac{3}{2} n$ and $(k)_{n} \geq k^{n}(n \in \mathbb{N})$ for $|z|<1$,

$$
\begin{aligned}
\left|T_{p, b, c}^{\prime}(z)-\frac{T_{p, b, c}(z)}{z}\right| & =\left|\sum_{n=2}^{\infty} \frac{\left(-\frac{c}{4}\right)^{n-1}}{\left(\frac{3}{2}\right)_{n-1}(k)_{n-1}}(n-1) a_{n} z^{n-1}\right| \\
& \leq \frac{2}{3} \sum_{n=2}^{\infty} n\left(\frac{|c|}{4 k}\right)^{n-1} \quad\left(\text { as }\left|a_{n}\right| \leq n \text { for } n \geq 2 \text { by Theorem } 1\right) \\
& =\frac{2}{3} \frac{|c|}{4 k}\left[\frac{1}{1-\frac{|c|}{4 k}}+\frac{1}{\left(1-\frac{|c|}{4 k}\right)^{2}}\right] \\
& =\frac{16 k|c|-2|c|^{2}}{3(4 k-|c|)^{2}} .
\end{aligned}
$$

Obviously, the restriction on $k$ is $k>\frac{|c|}{4}$. On the other hand, using $\left|z_{1}-z_{2}\right| \geq|| z_{1}|-| z_{2}||$, the inequalities $\left(\frac{3}{2}\right)_{n} \geq\left(\frac{3}{2}\right)^{n}$ and $(k)_{n} \geq k^{n}(n \in \mathbb{N})$ for $|z|<1$,

$$
\begin{aligned}
\left|\frac{T_{p, b, c}(z)}{z}\right| & =\left|1+\sum_{n=2}^{\infty} \frac{\left(-\frac{c}{4}\right)^{n-1}}{\left(\frac{3}{2}\right)_{n-1}(k)_{n-1}} a_{n} z^{n-1}\right| \\
& \geq 1-\sum_{n=2}^{\infty}(n+1)\left(\frac{|c|}{6 k}\right)^{n} \\
& =1-\frac{|c|}{6 k}\left[\frac{1}{1-\frac{|c|}{6 k}}+\frac{1}{\left(1-\frac{|c|}{6 k}\right)^{2}}\right] \\
& =\frac{2\left(18 k^{2}-12 k|c|+|c|^{2}\right)}{(6 k-|c|)^{2}}
\end{aligned}
$$


where the restriction on $k$ is $k>\frac{|c|}{3}\left(1+\frac{1}{\sqrt{2}}\right)$. Combining Equations (8) and (9) gives

$$
\begin{aligned}
\left|\frac{z T_{p, b, c}^{\prime}(z)}{T_{p, b, c}(z)}-1\right| & =\left|T_{p, b, c}^{\prime}(z)-\frac{T_{p, b, c}(z)}{z}\right|\left|\frac{z}{T_{p, b, c}(z)}\right| \\
& <\left(\frac{16 k|c|-2|c| c \mid}{3\left(4 k-\left.|c|\right|^{2}\right.}\right)\left(\frac{(6 k-|c|)^{2}}{2\left(18 k^{2}-12 k|c|+|c|^{2}\right)}\right) \\
& =\Psi(k,|c|) .
\end{aligned}
$$

Next, to determine the values of $k_{1}$ such that it satisfies

$$
\Psi\left(k_{1},|c|\right)<M,
$$

where $M$ is the solution of the equation $\cos (M)=M$, the inequality can be written as

$$
\begin{aligned}
& \Psi\left(k_{1},|c|\right)<M \\
& \Rightarrow\left(8 k_{1}|c|-|c|^{2}\right)\left(6 k_{1}-|c|\right)^{2}<3 M\left(4 k_{1}-|c|\right)^{2}\left(18 k_{1}^{2}-12 k_{1}|c|+|c|^{2}\right) \\
& \Rightarrow 288 k_{1}^{3}|c|-132 k_{1}^{2}|c|^{2}+20 k_{1}|c|^{3}+|c|^{4}<3 M\left(288 k_{1}^{4}-336 k_{1}^{3}|c|+130 k_{1}^{2}|c|^{2}-20 k_{1}|c|^{3}+|c|^{4}\right) \\
& \Rightarrow F\left(k_{1}\right)=M k_{1}^{4}-\frac{7 M+2}{6} k_{1}^{3}|c|+\frac{65 M+22}{144} k_{1}^{2}|c|^{2}-\frac{15 M+5}{216} k_{1}|c|^{3}+\frac{3 M+1}{864}|c|^{4}>0 .
\end{aligned}
$$

Using Proposition 3 to find $F(x)$,

$$
M x^{4}-\frac{7 M+2}{6} x^{3}|c|+\frac{65 M+22}{144} x^{2}|c|^{2}-\frac{15 M+5}{216} x|c|^{3}+\frac{3 M+1}{864}|c|^{4}=0 .
$$

Putting

$$
\begin{gathered}
a=-\frac{17 M^{2}+40 M+12}{288 M^{2}}|c|^{2}, \\
d=-\frac{4 M^{3}+25 M^{2}+20 M+4}{864 M^{3}}|c|^{4}, \\
\Delta_{0}=\frac{49 M^{2}+28 M+4}{20736}|c|^{4}, \\
\Delta_{1}=-\frac{343 M^{3}+2886 M^{2}+948 M+8}{1492992}|c|^{6}, \\
\Delta_{1}^{2}-4 \Delta_{0}^{3}=\frac{1728 M\left(1029 M^{4}+5113 M^{3}+3138 M^{2}+540 M+8\right)}{1492992^{2}}|c|^{12}, \\
Q=\frac{\alpha}{144}|c|^{2} \text { where } \alpha \text { is }(7), \\
S=\frac{\beta}{24 M}|c| \text { where } \beta \text { is }(6),
\end{gathered}
$$

and since $\cos (M)=M$ gives $M \approx 0.7390851332$, the zeros of $F$ are

$$
\left\{\begin{array}{l}
x_{1,2} \in \mathbb{C} \text { as }-4 S^{2}-2 a+\frac{d}{S}<0 \\
x_{3,4} \in \mathbb{R} \text { as }-4 S^{2}-2 a-\frac{d}{S}>0 .
\end{array}\right.
$$

The real roots $x_{3,4}$ are given by

$$
\begin{aligned}
x_{3,4} & =-\frac{s}{4 r}+S \pm \frac{1}{2} \sqrt{-4 S-2 a-\frac{d}{S}} \\
& =\frac{7 M+2+\beta \pm \sqrt{\frac{16 M^{3}+(17 \beta+100) M^{2}+(40 \beta+80) M+12 \beta+16-\beta^{3}}{\beta}}}{24 M}|c|,
\end{aligned}
$$


which upon simplification, results in the following approximation:

$$
\begin{gathered}
x_{3}=\frac{7 M+2+\beta-\sqrt{\frac{16 M^{3}+(17 \beta+100) M^{2}+(40 \beta+80) M+12 \beta+16-\beta^{3}}{\beta}}}{24 M}|c| \\
x_{4}=\frac{7 M+2+\beta+\sqrt{\frac{16 M^{3}+(17 \beta+100) M^{2}+(40 \beta+80) M+12 \beta+16-\beta^{3}}{\beta}}}{24 M}|c| \\
\approx 1.09813352|c| .
\end{gathered}
$$

Thus, it can be concluded that $F\left(k_{1}\right)>0$ for $k_{1}<x_{3}$ or $k_{1}>x_{4}$. Since $k>\frac{|c|}{3}\left(1+\frac{1}{\sqrt{2}}\right)$, then

$$
k>x_{4}=\frac{7 M+2+\beta+\sqrt{\frac{16 M^{3}+(17 \beta+100) M^{2}+(40 \beta+80) M+12 \beta+16-\beta^{3}}{\beta}}}{24 M}|c|,
$$

is the range of values of $k_{1}$ that satisfies Equation (11). As $T_{p, b, c}$ is an analytic function, then, by Lemma $1, \Re\left\{T_{p, b, c}(z)\right\}>0$ for all $z \in U$.

Remark 1. Obviously from Lemma 2, with $c$ and $k$ satisfying the constraints given in Theorem 2, the function $T_{p, b, c}$ is univalent in $U$.

Theorem 3. Let $f \in \mathcal{A}$ and $T_{p, b, c}(z)$ be defined by Equation (5). If $p, b, c, \in \mathbb{C}, k_{2}=p+\frac{b+2}{2}$ and

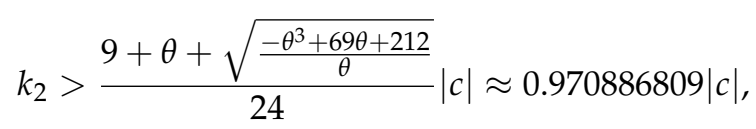

where

$$
\theta=\sqrt{23+\sqrt[3]{-155+16 \sqrt{91}}+\frac{9}{\sqrt[3]{-155+16 \sqrt{91}}}} \approx 3.862149964,
$$

then $T_{p, b, c}$ is starlike in $U$.

Proof. Suppose $f \in \mathcal{A}$. Similar to the previous result, the aim is to seek constraints on $k_{2}$ such that $T_{p, b, c}$ is starlike in $U$. Hence, replacing $k_{1}$ with $k_{2}$ and $M$ with 1 in Equation (11) gives the following:

$$
\begin{aligned}
& \Psi\left(k_{2},|c|\right)<1 \\
& \Rightarrow 4|c|^{2}-80 k_{2}|c|^{3}+522 k_{2}^{2}|c|^{2}-1296 k_{2}^{3}|c|+864 k_{2}^{4}>0 \\
& \Rightarrow G\left(k_{2}\right)=k_{2}^{4}-\frac{3}{2} k_{2}^{3}|c|+\frac{29}{48} k_{2}^{2}|c|^{2}-\frac{5}{54} k_{2}|c|^{3}+\frac{1}{216}|c|^{4}>0
\end{aligned}
$$

Once again, using Proposition 3 to find the zeros of $G(y)$,

$$
y^{4}-\frac{3}{2} y^{3}|c|+\frac{29}{48} y^{2}|c|^{2}-\frac{5}{54} y|c|^{3}+\frac{1}{216}|c|^{4}=0 .
$$


Putting

$$
\begin{gathered}
a=-\frac{23}{96}|c|^{2}, \\
d=-\frac{56}{864}|c|^{4}, \\
\Delta_{0}=\frac{1}{256}|c|^{4}, \\
\Delta_{1}=-\frac{155}{55296}|c|^{6}, \\
\Delta_{1}^{2}-4 \Delta_{0}^{3}=\frac{91}{11943936}|c|^{12}, \\
Q=\sqrt[3]{\frac{-155+16 \sqrt{91}}{48}|c|^{2},} \\
S=\frac{\theta}{24}|c| \text { where } \theta \text { is (13), }
\end{gathered}
$$

the zeros of $G$ are

$$
\left\{\begin{array}{l}
y_{1,2} \in \mathbb{C} \text { as }-4 S^{2}-2 a+\frac{d}{S}<0 \\
y_{3,4} \in \mathbb{R} \text { as }-4 S^{2}-2 a-\frac{d}{S}>0
\end{array}\right.
$$

The real roots $y_{3,4}$ are given by

$$
y_{3,4}=-\frac{s}{4 r}+S \pm \frac{1}{2} \sqrt{-4 S-2 a-\frac{d}{S}}=\frac{9+\theta \pm \sqrt{\frac{-\theta^{3}+69 \theta+212}{\theta}}}{24}|c|,
$$

which, upon simplification, results in the following approximation:

$$
\begin{gathered}
y_{3}=\frac{9+\theta-\sqrt{\frac{-\theta^{3}+69 \theta+212}{\theta}}|c| \approx 0.00958649|c|,}{24} \mid \\
y_{4}=\frac{9+\theta+\sqrt{\frac{-\theta^{3}+69 \theta+212}{\theta}}}{24}|c| \approx 0.970886809|c| .
\end{gathered}
$$

Thus, it can be concluded that $G\left(k_{2}\right)>0$ for $k_{2}<y_{3}$ or $k_{2}>y_{4}$. Since $k>\frac{|c|}{3}\left(1+\frac{1}{\sqrt{2}}\right)$, then

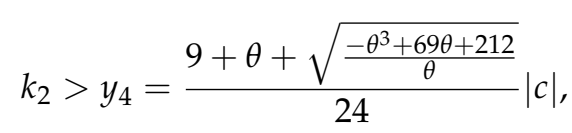

is the range of values of $k_{2}$ that satisfies Equation (14) which, in turn, implies that $T_{p, b, c}$ is starlike in $U$ by Lemma 3 when $\alpha=\beta=0$.

Theorem 4. Let $f \in \mathcal{A}$ and $T_{p, b, c}(z)$ defined by Equation (5). If $p, b, c \in \mathbb{C}, k_{3}=p+\frac{b+2}{2}$ and $k_{3}>$ $\frac{13+\sqrt{130}}{12}|c|$, then $T_{p, b, c}(z)$ is convex in $U$. 
Proof. Suppose $f \in \mathcal{A}$. Using $\left|z_{1}+z_{2}\right| \leq\left|z_{1}\right|+\left|z_{2}\right|$, the inequalities $\left(\frac{3}{2}\right)_{n}>\frac{n(n+1)}{2}$ and $(k)_{n} \geq k^{n}$ $(n \in \mathbb{N})$ for $|z|<1$,

$$
\begin{aligned}
\left|z T_{p, b, c}^{\prime \prime}(z)\right| & =\left|\sum_{n=2}^{\infty} \frac{\left(-\frac{c}{4}\right)^{n-1}}{\left(\frac{3}{2}\right)_{n-1}(k)_{n-1}} n(n-1) a_{n} z^{n-1}\right| \\
& \leq 2 \sum_{n=2}^{\infty} n\left(\frac{|c|}{4 k}\right)^{n-1}\left(\text { as }\left|a_{n}\right| \leq n \text { for } n \geq 2 \text { by Theorem } 1\right) \\
& =2\left(\frac{|c|}{4 k}\right)\left[\frac{1}{1-\frac{|c|}{4 k}}+\frac{1}{\left(1-\frac{|c|}{4 k}\right)^{2}}\right] \\
& =\frac{16 k|c|-2|c|^{2}}{(4 k-|c|)^{2}} .
\end{aligned}
$$

Obviously, the restriction on $k$ is $k>\frac{|c|}{4}$. Using $\left|z_{1}-z_{2}\right| \geq|| z_{1}|-| z_{2}||$, the inequalities $\left(\frac{3}{2}\right)_{n}>$ $\frac{3(n+1)}{4}$ and $(k)_{n} \geq k^{n}(n \in \mathbb{N})$ for $|z|<1$,

$$
\begin{aligned}
\left|T_{p, b, c}^{\prime}(z)\right| & =\left|1+\sum_{n=2}^{\infty} \frac{\left(-\frac{c}{4}\right)^{n-1}}{\left(\frac{3}{2}\right)_{n}(k)_{n-1}} n a_{n} z^{n-1}\right| \\
& \geq 1-\frac{4}{3} \sum_{n=2}^{\infty} n\left(\frac{|c|}{4 k}\right)^{n-1} \\
& =1-\frac{4}{3}\left(\frac{|c|}{4 k}\right)\left[\frac{1}{1-\frac{|c|}{4 k}}+\frac{1}{\left(1-\frac{|c|}{4 k}\right)^{2}}\right] \\
& =\frac{48 k^{2}-56 k|c|+7|c|^{2}}{3(4 k-|c|)^{2}},
\end{aligned}
$$

where the restriction on $k$ is $k>\frac{7+2 \sqrt{7}}{12}|c|$. Comparing Equations (16) and (17) gives the following:

$$
\left|\frac{z T_{p, b, c}^{\prime \prime}(z)}{T_{p, b, c}^{\prime}(z)}\right|=\left|z T_{p, b, c}^{\prime \prime}(z)\right|\left|\frac{1}{T_{p, b, c}^{\prime}(z)}\right|<\frac{48 k|c|-6|c|^{2}}{48 k^{2}-56 k|c|+7|c|^{2}} .
$$

The next step is to determine the values of $k_{3}$ such that it satisfies the following:

$$
\frac{48 k|c|-6|c|^{2}}{48 k^{2}-56 k|c|+7|c|^{2}}<1
$$

From Equation (17),

$$
\begin{aligned}
& \frac{48 k_{3}|c|-6|c|^{2}}{48 k_{3}^{2}-56 k_{3}|c|+7|c|^{2}}<1 \\
& \Rightarrow 48 k_{3}|c|-6|c|^{2}<48 k_{3}^{2}-56 k_{3}|c|+7|c|^{2} \\
& \Rightarrow 48 k_{3}^{2}-104 k_{3}|c|+13|c|^{2}>0 \\
& \Rightarrow k_{3}^{2}-\frac{13}{6} k_{3}|c|+\frac{13}{48}|c|^{2}>0 \\
& \Rightarrow k_{3}<\frac{13-\sqrt{130}}{12}|c| \approx 0.133187|c| \text { OR } k_{3}>\frac{13+\sqrt{130}}{12}|c| \approx 2.03345|c| .
\end{aligned}
$$

Since $k>\frac{7+2 \sqrt{7}}{12}|c|$, then $k_{3}>\frac{13+\sqrt{130}}{12}|c|$ is the range of values of $k_{3}$ such that it satisfies Equation (17). Since the range of values of $k_{3}$ satisfies the condition in Theorem 3, then $z T_{p, b, c}^{\prime}$ is starlike in $U$ which, in turn, implies that $T_{p, b, c}$ is convex in $U$ by the Alexander theorem.

\section{Conclusions}

In summary, the bounds on $k$ for function $T_{p, b, c}$ to be univalent, starlike, and convex were obtained. Specifically, for $k=k_{1}>1.098143352|c|, T_{p, b, c}$ is univalent; for $k=k_{2}>0.970886809|c|, T_{p, b, c}$ is starlike; and for $k=k_{3}>2.03345|c|, T_{p, b, c}$ is convex. The bounds obtained for convexity and starlikeness of the 
function $T_{p, b, c}$ are in agreement since $k_{2}<k_{3}$ and all convex functions are starlike. Secondly, the same goes for the function to be univalent and convex since $k_{1}<k_{3}$ and all convex functions are univalent for $z \in U$. However, since the bounds obtained in $k_{1}$ and $k_{2}$ are not necessarily sharp, the relationship between $k_{1}$ and $k_{2}$ does not imply the outcome that all starlike functions are univalent in the unit disk. This is probably due to the fact that the approach in establishing Theorem 3 used Lemma 3 (a necessary condition) as opposed to using Proposition 1 (necessary and sufficient). This allows for further research to explore better methods, such as using Proposition 1, to achieve better bounds. Nevertheless, the results attained in this paper are in adherence with the results in Reference [26]. Conducting further research in the future will hopefully produce a sharper result.

Author Contributions: All authors contributed equally in writing this article. All authors read and approved the final manuscript.

Funding: This research received no external funding.

Conflicts of Interest: The authors declare no conflicts of interest.

\section{References}

1. Srivastava, H.M.; Manocha, H.L. A Treatise on Generating Functions; John Wiley \& Sons: New York, NY, USA, 1984.

2. Cesarano, C. Some Special Classes of Bessel Functions and Modified Humbert Functions. Int. J. Pure Appl. Math. 2016, 111, 589-604.

3. Balasubramanian, R.; Ponnusamy, S.; Prabhakarna, D.J. Convexity of Integral Transforms and Functional Spaces. Integr. Transforms Spec. Funct. 2007, 18, 1-14. [CrossRef]

4. Miller, S.S.; Mocanu, P.T. Univalence of Gaussian and Confluent Hypergeometric Functions. Proc. Am. Math. Soc. 1990, 110, 333-342. [CrossRef]

5. Ponnusamy, S.; Ronning, F. Geometric Properties for Convolutions of Hypergeometric Functions and Functions with the Derivative in a Halfplane. Integr. Transforms Spec. Funct. 1999, 8, 121-138. [CrossRef]

6. Ponnusamy, S.; Vuorinen, M. Univalence and Convexity Properties for Confluent Hypergeometric Functions. Complex Var. Theory Appl. 1998, 36, 73-97. [CrossRef]

7. Ponnusamy, S.; Vuorinen, M. Univalence and Convexity Properties for Gaussian Hypergeometric Functions. Rocky Mt. J. Math. 2001, 31, 327-353. [CrossRef]

8. Ponnusamy, S.; Singh, V.; Vasundra, P. Starlikeness and Convexity of an Integral Transform. Integr. Transforms Spec. Funct. 2004, 15, 267-280. [CrossRef]

9. Baricz, A.; Ponnusamy, S. Integral Transforms and Special Functions. Integr. Transforms Spec. Funct. 2010, 21, 641-653. [CrossRef]

10. Küstner, R. On the Order of Starlikeness of the Shifted Gauss Hypergeometric Functions. J. Math. Anal. Appl. 2007, 334, 1363-1385. [CrossRef]

11. Ruscheweyh, St.; Singh, V. On the Order of Starlikness of Hypergeometric Functions. J. Math. Anal. Appl. 1986, 113, 1-11. [CrossRef]

12. Silverman, H. Starlike and Convexity Properties for Hypergeometric Functions. J. Math. Anal. Appl. 1993, 172, 574-581. [CrossRef]

13. Baricz, A. Geometric Properties of Generalized Bessel Functions. Publ. Math. Debreceen 2008, 73, $155-178$.

14. Deniz, E.; Orhan, H.; Srivastava, H.M. Some Sufficient Conditions for Univalence of Certain Families of Integral Operators Involving Generalized Bessel Functions. Taiwanese J. Math. 2011, 15, 883-917.

15. Deniz, E. Convexity of Integral Operators Involving Generalized Bessel Fucntions. Integr. Transforms Spec. Funct. 2013, 24, 201-216. [CrossRef]

16. Owa, S.; Srivastava, H. Univalent and Starlike Generalized Hypergeometric Functions. Can. J. Math. 1987, 67, 199-211. [CrossRef]

17. Răducanu, D.; Srivastava, H.M. A New Class of Analytic Functions Defined by means of a Convolution Operator Involving the Hurwitz-Lerch Zeta Function. Integr. Transforms Spec. Funct. 2007, 18, 933-943. [CrossRef]

18. Selinger, V. Geometric Properties of Normalized Bessel Functions. Pure Math. Appl. 1995, 6, $273-277$. 
19. Srivastava, H.M.; Yang, D.G.; Xu, N.E. Subordinations for Multivalent Analytic Functions Associated with Dziok-Srivastava Operator. Integr. Transforms Spec. Funct. 2009, 20, 581-606. [CrossRef]

20. Srivastava, H.M. Generalized Hypergeometric Functions and Associated Families of k-uniformity Convex and k-starlike Functions. Gen. Math. 2007, 15, 201-226.

21. Duren, P.L. Univalent Functions; Springer-Verlak: New York, NY, USA, 1983.

22. Goodman, A.W. Univalent Functions; Mariner Publication Company: Tanpa, FL, USA, 1983.

23. Alexander, J.W. Functions which Map the Interior of the Unit Circle upon Simple Regions. Ann. Math. 1915, 17, 12-22. [CrossRef]

24. Zhang, S.; Jin, J. Computation of Special Functions; John \& Sons Inc.: New York, NY, USA, 1996.

25. Watson, G.N. A Treatise on the Theory of Bessel Functions; University Press-Cambridge: Cambridge, UK, 1922.

26. Orhan, H.; Yagmur, N. Geometric Properties of Generalized Struve Functions. Ann. Alexandru Ioan Cuza Univ. Math. 2014. [CrossRef]

27. Yagmur, N.; Orhan, H. Starlikeness and Convexity of Generalized Struve Functions. Abstr. Appl. Anal. 2013, 6. [CrossRef]

28. Yagmur, N.; Orhan, H. Hardy Space of Generalized Struve Functions. Complex Var. Elliptic Equ. 2014, 59, 929-937. [CrossRef]

29. Raza, M.; Yagmur, N. Some Properties of a Class of Analytic Functions defined by Geometric Struve Functions. Turkish J. Math. 2015, 39, 931-944. [CrossRef]

30. Branges, L. A Proof of the Bieberbach Conjecture. Acta Math. 1985, 154, 137-152. [CrossRef]

31. Xanthopoulos, X. Subclasses of Starlike Functions with $\Re\left\{f^{\prime}(z)\right\}>0$. Studia Univ. Babeş-Boylai Math. 1993, $38,39-47$.

32. MacGregor, T. Functions whose Derivative has a Positive Real Part. Trans. Am. Math. Soc. 1962, 104, $532-537$. [CrossRef]

33. Cardano, G. Artis Magnae, Sive de Regulis Algebraicis Liber Unus; Franco Angeli: Milan, Italy, 1545.

(C) 2018 by the authors. Licensee MDPI, Basel, Switzerland. This article is an open access article distributed under the terms and conditions of the Creative Commons Attribution (CC BY) license (http:// creativecommons.org/licenses/by/4.0/). 